\title{
Nuances in the Treatment of Malignant Tumors of the Clival and Petroclival Region
}

\author{
Ahmed Mohyeldin ${ }^{1}$ Daniel M. Prevedello ${ }^{1,2}$ Ali O. Jamshidi ${ }^{1} \quad$ Leo F.S. Ditzel Filho ${ }^{1}$ Ricardo L. Carrau ${ }^{1,2}$ \\ 1 Department of Neurological Surgery, The Ohio State University \\ Wexner Medical Center, Columbus, Ohio, United States \\ 2 Department of Otolaryngology-Head and Neck Surgery, The Ohio State \\ University Wexner Medical Center, Columbus, Ohio, United States

\begin{abstract}
Address for correspondence Daniel M. Prevedello, MD, The Ohio State University Wexner Medical Center, 410 W. 10th Avenue, N-1049 Doan Hall, Department of Neurological Surgery, Columbus, OH 43210, United States (e-mail: Daniel.Prevedello@osumc.edu).
\end{abstract}

Int Arch Otorhinolaryngol 2014;18:S157-S172.

\begin{abstract}
Introduction Malignancies of the clivus and petroclival region are mainly chordomas and chondrosarcomas. Although a spectrum of malignancies may present in this area, a finite group of commonly encountered malignant pathologies will be the focus of this review, as they are recognized to be formidable pathologies due to adjacent critical neurovascular structures and challenging surgical approaches.

Objectives The objective is to review the literature regarding medical and surgical management of malignant tumors of the clival and petroclival region with a focus on clinical presentation, diagnostic identification, and associated adjuvant therapies. We will also discuss our current treatment paradigm using endoscopic, open, and combined approaches to the skull base.

\section{Keywords}

- skull base

- malignancy

- clival

- petroclival

- endoscopic endonasal

- craniotomy

- chordoma

- chondrosarcoma

- plasmacytoma

Data Synthesis A literature review was conducted, searching for basic science and clinical evidence from PubMed, Medline, and the Cochrane Database. The selection criteria encompassed original articles including data from both basic science and clinical literature, case series, case reports, and review articles on the etiology, diagnosis, treatment, and management of skull base malignancies in the clival and petroclival region.

Conclusions The management of petroclival malignancies requires a multidisciplinary team to deliver the most complete surgical resection, with minimal morbidity, followed by appropriate adjuvant therapy. We advocate the combination of endoscopic and open approaches (traditional or minimally invasive) as required by the particular tumor followed by radiation therapy to optimize oncologic outcomes.
\end{abstract}

\section{Introduction}

Management of malignant petroclival tumors requires a multidisciplinary team. A family doctor, neurologist, or ophthalmologist often identifies the tumors upon the patient's initial presentation, as the most common presenting symptom is double vision. This is often caused by impaired function of cranial nerve $\mathrm{VI}$, which passes immediately posterior to the petroclival synchondrosis. Their surgical management is challenging, compelling the involvement of multiple specialties to achieve satisfactory tumor resections with negligible or no morbidity. Surgical planning starts with neuroradiologists studying and delineating the intrinsic characteristics of the tumor and its relationship with important neurovascular structures. Medical specialties, including internal medicine and endocrinology, also should evaluate the patient preoperatively to optimize the patient's condition for surgery. Otolaryngologists, head and neck surgeons, neuroophthalmologists, and neurosurgeons represent the core surgical team. Ideally, the surgical team should be versed in a variety of open, endoscopic, and microscopic surgical 
approaches to the skull base. This latter parameter is important, as the surgical strategy must be individualized for each patient, customizing the approach (or approaches) to produce the least disruption of normal tissue, therefore preserving or restoring function. Furthermore, the surgical management of these tumors requires in-depth anatomical knowledge and team experience.

Following surgery, another team of professionals focuses on the patient's recovery and rehabilitation. Neurointensivists and rehabilitation physicians play a key role during this period, as well as nurses, physiotherapists, speech pathologists, occupational therapists, and social workers. Once the patient has recovered from the surgical stress, medical and radiation oncologists become central, defining and implementing the best strategy for long-term disease control.

\section{Anatomy of the Skull Base and the Margins of the Clivus and Petroclival Region}

The clivus, which is Latin for "slope," is part of the occipital bone. It extends inferiorly and obliquely from the dorsum sellae toward the foramen magnum. The most superior and anterior margins of the clivus form a joint with the dorsum sellae and define the anterior and most superior margins of the posterior fossa. The clivus has a unique anatomical relationship with the sphenoid bone in that it lies posterior and extends inferior to the sphenoid sinus, making it surgically accessible through transsphenoidal and nasopharyngeal approaches.

The petrous portion of the temporal bone is wedged in the middle of the skull base bordered anteriorly by the squamous part of the temporal bone and sphenoid bone and bordered posteriorly by the occipital bone. Its anterior surface makes up the margins of the middle cranial fossa, and its posterior surface makes up the margins of the posterior fossa.

Tumors expanding from the clival and petroclival regions can affect several key neurovascular elements, including the anterolateral surface of the pons, the basilar artery and its branches, and cranial nerves III through XII. The clival dura is also singular due to its double-layer configuration, the rich basilar venous plexus, and the abundant surrounding sinusoidal venous system. Tumors in this region can displace, engulf, or even infiltrate these structures, which manifest with critical clinical patterns that should be recognized preoperatively to plan the most appropriate surgical approach.

The most commonly affected cranial nerve is the sixth, which shares an intimate relationship with the petroclival synchondrosis. Cranial nerve VI travels through the subarachnoid space and pierces the meningeal layer of dura immediately behind the petroclival synchondrosis and then travels in the intradural space, then between the meningeal layer and the petroclival periosteum, in a superolateral orientation toward Dorello's canal. In this area, cranial nerve VI shares the space with the inferior petrosal sinus and is most vulner- able to lesions growing from the clivus and petroclival regions.

\section{Review of the Literature}

\section{Malignant Pathologies of the Clival and Petroclival Regions}

\section{Chordoma}

Chordoma is a rare cancer that accounts for 1 to $4 \%$ of all bone malignancies. ${ }^{1}$ Thought to arise from transformed remnants of notochord, chordomas have a mysterious predilection to present in the axial skeleton, most commonly at the sacrum and the clivus. Recent evidence suggests that chordomas distribute equally among the skull base, mobile spine, and sacrum. ${ }^{2} \mathrm{~A}$ comprehensive study aimed at evaluating the frequency of chordoma at different anatomic locations found that $32 \%$ were cranial, $32.8 \%$ were spinal, and $29.2 \%$ were sacral. $^{2}$

Recent genetic analysis of chordoma using high-resolution array CGH in tumor samples from patients with familial chordoma revealed unique duplications in the 6 q27 region. ${ }^{3}$ Interestingly, the duplicated region only contained the $\mathrm{T}$ (brachyury) gene, which was previously found to be uniquely overexpressed in almost all sporadic chordomas compared with other bone or cartilaginous lesions. ${ }^{4,5}$ Brachyury regulates several compelling stem cell genes and has recently been implicated in promoting epithelial-mesenchymal transition in other human carcinomas. ${ }^{6}$ Although it is still not clear what role brachyury plays in the pathogenesis of chordomas, the identification of the duplication and the remarkable overexpression seen in samples suggest that it may be a critical molecular driver in the initiation and propagation of this cancer.

Although considered to be a histologically low-grade malignant neoplasm, chordomas have a poor prognosis with notorious local recurrence rates that make their clinical progression very similar to that of malignant tumors. ${ }^{7,8}$ Population-based studies using the Surveillance, Epidemiology and End Results (SEER) database suggest an incidence rate of 0.08 per 100,000 , with predominance in men over women and very low incidence in patients below 40 years of age. ${ }^{2}$ In fact, chordomas in children and adolescents account for $<5 \%$ of all chordoma cases. Its peak incidence is between 50 and 60 years of age. ${ }^{2,9}$ The indolent and slow-growing features that commonly characterize chordomas contribute to their frequently asymptomatic clinical presentation; thus, they often remain silent until late stages of the disease. By this time, patients present with focal neurologic deficits (e.g., cranial nerve VI palsy) often in addition to headaches and neck pain and even endocrinopathies due to local mass effect. ${ }^{10}$

Because of the notochordal origin of these tumors, chordomas are midline entities; thus, in contrast to chondrosarcomas, they tend to grow from midline, expanding posteriorly and laterally. They often appear on radiographs as destructive bone lesions associated with a large soft tissue mass. - Fig. 1 demonstrates the case of a 21-year-old patient who presented late in the course of the disease with diplopia, dysphagia, and left upper extremity weakness due to the severe brainstem compression. Calcifications and bony 

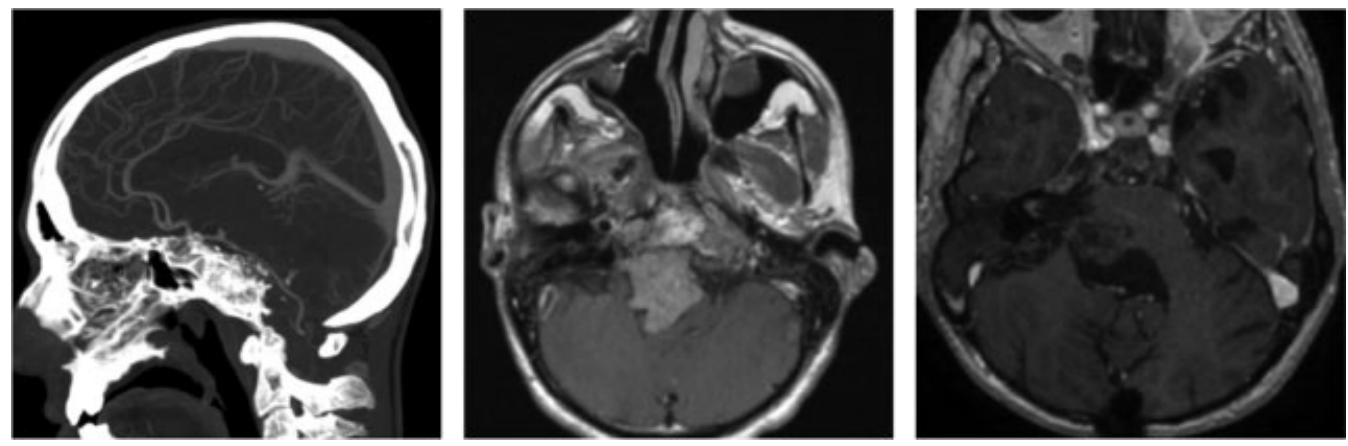

Fig. 1 Clival chordoma in a 21-year-old man, who presented with a large compressive midline lesion and diplopia, dysphagia, and left upper extremity weakness. (Left) Sagittal computed tomography and arterial angiography scan demonstrating a large expansile lytic mass in the clivus with compression of important surrounding neurovascular structures and brainstem. (Middle) Magnetic resonance imaging (MRI) of postcontrast T1-weighted sequence axial scans of a heterogeneously enhancing chordoma with significant mass effect. (Right) Postoperative MRI postcontrast T1-weighted scans showing extensive resection and decompression of around brainstem.

expansion are frequent features on magnetic resonance imaging (MRI), and characteristically chordomas appear isointense or hypointense on T1-weighted images and hyperintense on T2-weighted images and heterogeneously enhance with gadolinium. ${ }^{11}$ Furthermore, chordomas demonstrate reduced or normal uptake of radioisotope on bone scan when juxtaposed to other bone tumors. ${ }^{12}$

Chordomas are relatively insensitive to both radiotherapy and chemotherapy; as a result, surgery is the preferred treatment modality for this tumor. The use of radiotherapy as a primary or adjuvant treatment in various treatment paradigms for chordoma has been a subject of intense debate. Unfortunately, stand-alone radiotherapy has been ineffective, even when coupled with debulking or palliative decompression. ${ }^{13}$-Fig. 2 illustrates the case of patient who was involved in a motor vehicle accident and a subtle abnormality near his clivus was found. Follow-up imaging revealed a small-interval growth of the median clival mass, which on MRI appeared consistent with chordoma. The patient ultimately presented with paralysis of the abducens nerve on the left. Given the progression and new diplopia, an endoscopic endonasal approach was undertaken, achieving a gross total resection. Subsequent to surgery, the patient's diplopia improved and he was referred to proton beam therapy after pathology confirmed the diagnosis of chordoma.

Advances in radiation technology with the introduction of hadrons (i.e., high-dose protons or charged particles
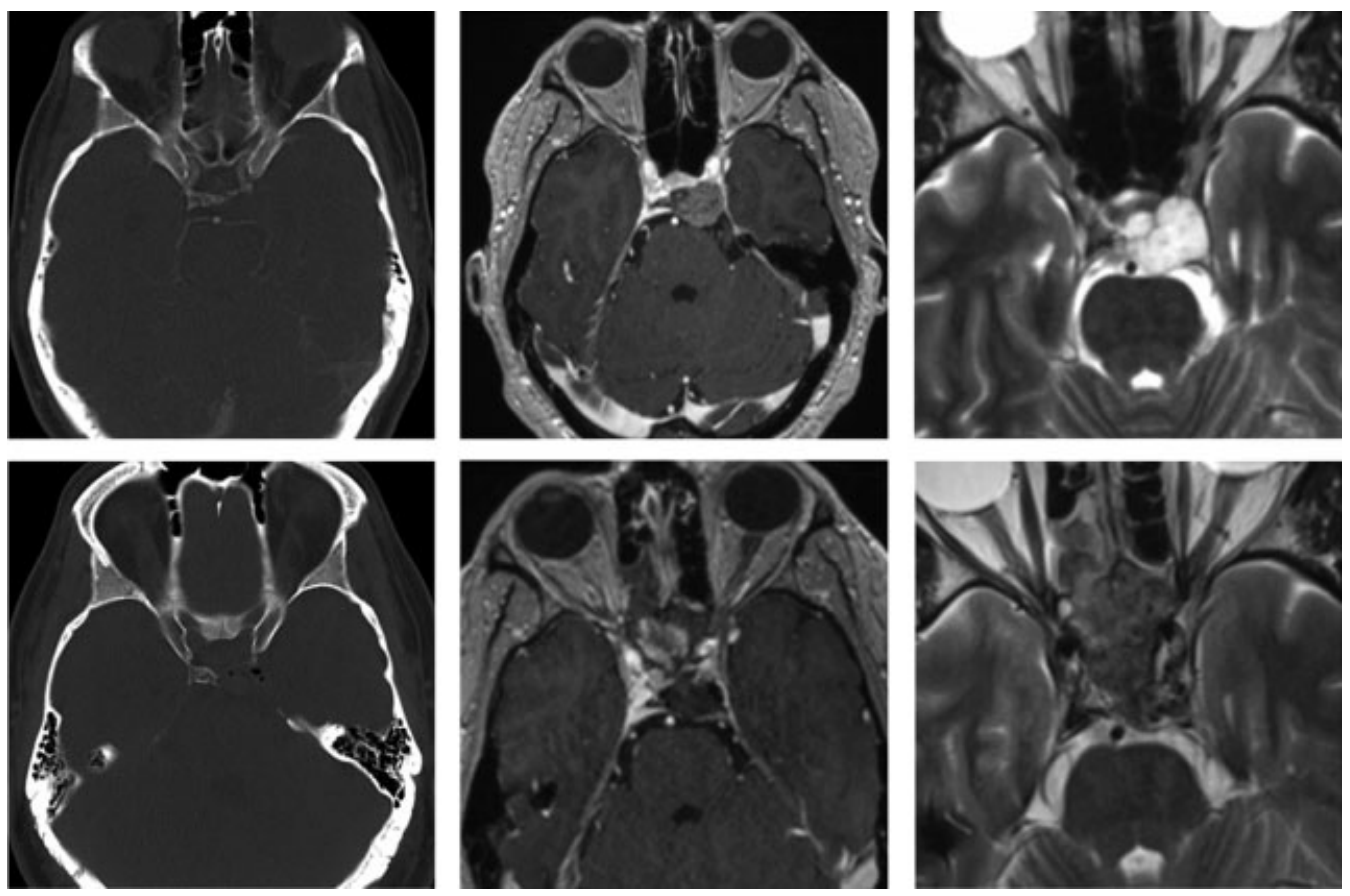

Fig. 2 Petroclival midline lytic mass incidentally discovered on computed tomography scan (CT) after a 38-year-old man was involved in a motor vehicle accident (top left). Follow-up scans with magnetic resonance imaging (MRI) revealed interval growth of the midline lesion and an enhancing pattern with a midline location consistent with chordoma (top middle). T2-weighted sequences revealed a hyperintense mass also consistent with chordoma (top right). Aggressive gross total resection evidenced by postoperative CT and MRI scan (bottom left, middle, and right) was followed by proton beam therapy. 
including carbon ions, helium, or neon) have facilitated the delivery of higher doses of radiation to the target volume with minimal surrounding tissue injury and improved radiobiological effect. ${ }^{14-16}$ Adjuvant proton beam therapy (PBT) after gross total resection is currently the accepted treatment standard in the management of chordoma, and it is the strategy currently favored by the authors.

The literature is scattered with various cases series reporting variable survival rates on small numbers of patients. However, the most comprehensive incidence and survival analysis involve 400 cases from the SEER database, which demonstrated a median survival of 6.29 years with 5-, 10-, and 20-year survival rates precipitously dropping to 67.6, 39.9 , and $13.1 \%$ across all ethnic groups and genders, thus underscoring the dismal prognosis of this disease. ${ }^{2}$ - Fig. 3 illustrates the aggressive biology of chordoma, demonstrating the progressive recurrence of a clival chordoma that was thoroughly resected and treated with adjuvant PBT. Despite the highest standard of care, local spread down the cervical spine led to cervicomedullary compression, paralysis, and eventual death of the patient.

\section{Chondrosarcoma}

Chondrosarcoma is also a rare primary bone neoplasm with an estimated incidence of 1 in 200,000. Chondrosarcomas account for nearly $6 \%$ of all skull base tumors and are more commonly found in the axial skeleton over the appendicular skeleton, making their presentation in the skull base a truly rare event $(\sim 1 \%) .{ }^{17}$ Radiographically, chondrosarcoma, like chordoma, appears as a destructive, expansile, mineralized mass on computed tomography (CT) that invades bone and extends into soft tissues. MRI often shows a moderate to intensely enhancing mass with a lobular structure of high signal intensity on T2-weighted images and with strong peripheral rim and septal enhancement patterns. ${ }^{18}$ However, the fact that chondrosarcomas originate from the petroclival synchondrosis distinguishes them from chordomas. They are paramedian lesions that often expand not only into the clivus and sphenoid sinus but also into the middle and posterior cranial fossae, as well as the upper cervical area (-Fig. 4).

Classically, chordomas were pathologically identified by their physaliferous features and immunoreaction for S-100 and epithelial markers such as EMA and cytokeratins. ${ }^{19-21}$ However, until recently, distinguishing between chondroid chordomas and chondrosarcomas was a diagnostic challenge because of their shared S-100 immunoreactivity and often difficult to interpret cytokeratin expression on small biopsies. $^{5,22}$ Several groups have postulated that the notochord developmental transcription factor brachyury, found to be overexpressed in nearly all chordomas, may serve as a discriminating novel biomarker to distinguish chordoma from chondrosarcoma. $4,5,8,22-24$

The majority of cranial chondrosarcomas are low-grade, slow-growing indolent tumors that have the capacity to locally engulf critical neurovascular structures. They can cause mass effect on the surrounding brainstem and can extend as far lateral as the temporal lobe superiorly and the jugular foramen inferiorly. As mentioned previously, their predilection to occur more laterally in the skull base when compared with chordomas is thought to be a consequence of their cell of origin. Chondrosarcomas are thought to derive from sporadic, malignant transformation of remnants of chondrocytes within the synchondroses of the skull base. As a result, they are often found in the paraclival region arising from the sphenopetrosal, petro-occipital (petroclival), or spheno-occipital synchondroses. - Fig. 4 shows the MRIs of a 47-year-old patient who presented with diplopia and headaches and was found to be harboring a right petroclival mass with involvement of the clivus and considerable mass effect on the brainstem and temporal lobe (note the laterality of the tumor compared with the midline predilection often seen with chordomas in - Figs. 1 and 2). Several case series that have analyzed chondrosarcoma patients found that the most common presenting symptoms were headache and diplopia. Cranial nerve VI is most commonly affected because of its superolateral projection toward to the petroclival synchondrosis and because of its course through the Dorello canal. ${ }^{25}$
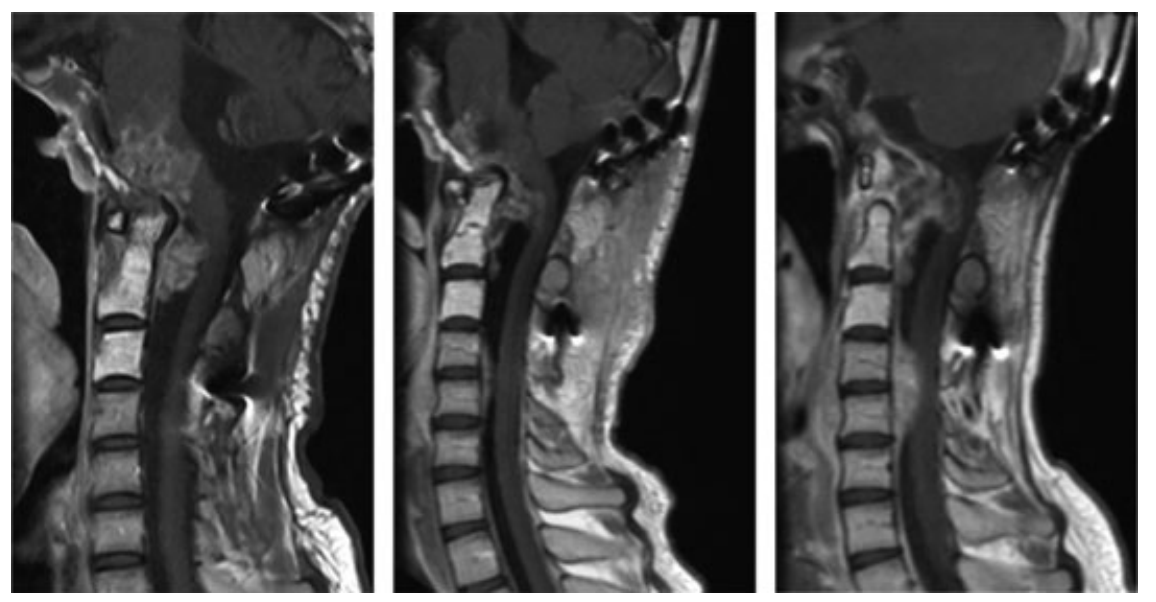

Fig. 3 Progressive recurrence of a clival chordoma that had been resected and treated with proton beam therapy. From left to right progressive recurrence over a 3-year period of local spread down the cervical spine inevitably leading to spinal cord compression, paralysis, and eventually death. 

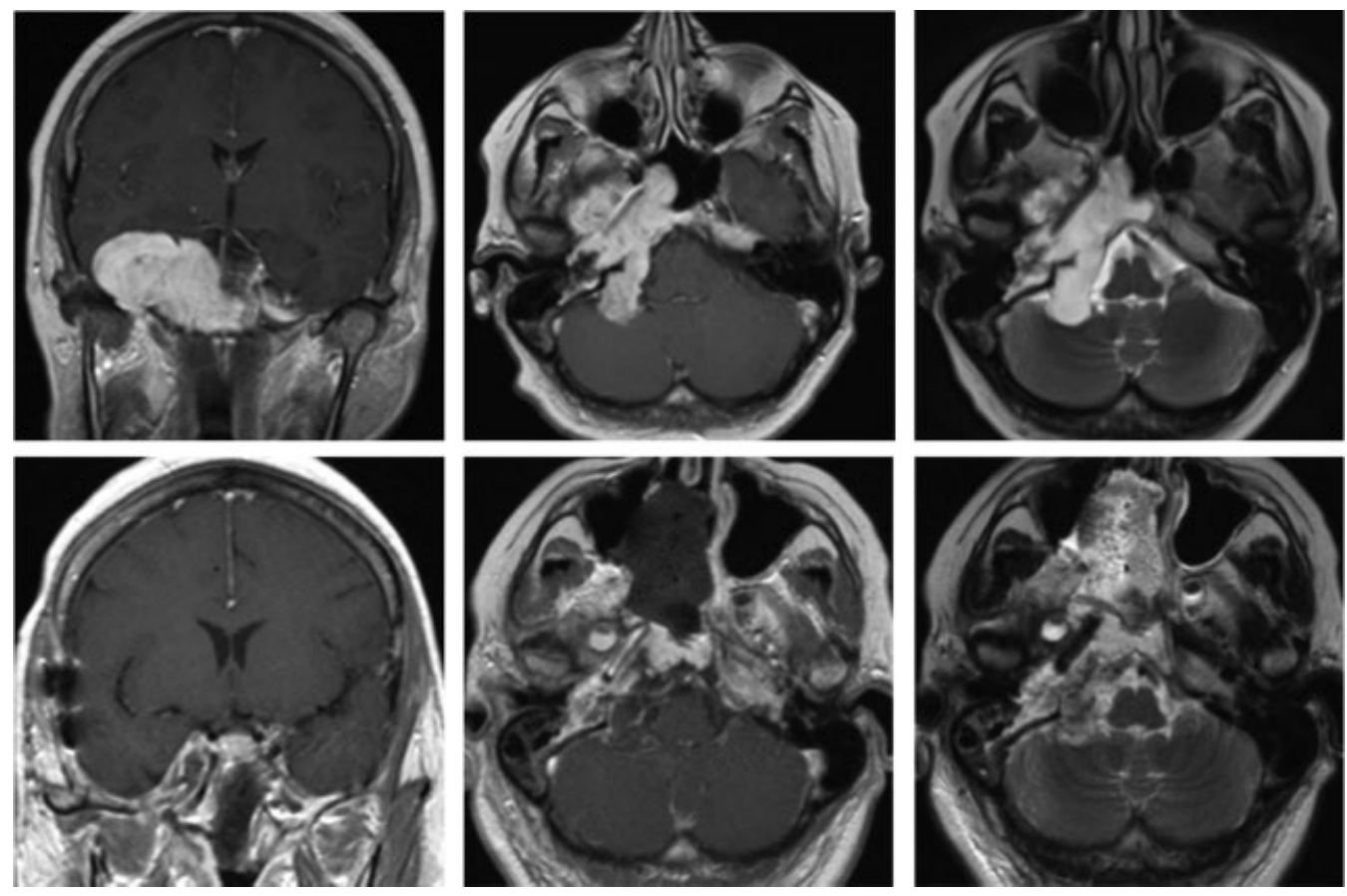

Fig. 4 Right-sided petroclival chondrosarcoma in a 47-year-old woman who presented with diplopia, headaches, and nausea/vomiting. Magnetic resonance imaging (MRI) revealed an enhancing mass at the right petrous apex with involvement of the clivus and significant temporal brain compression. (Top left) coronal MRI T1-weighted postcontrast scans demonstrating temporal lobe compression. (Top middle) axial MRI T1weighted postcontrast scans. (Top right) T2-weighted sequence scans demonstrating hyperintense lateral paraclival mass with posterior fossa compression. (Bottom) Postoperative MRI scans in matching order as the top row. Gross total resection was achieved via an endoscopic transsphenoidal surgical resection of the lesion, which had followed a subtemporal craniotomy for debulking performed previously elsewhere.

Population-based studies utilizing the SEER database suggest that histologic subtype and tumor grade are important prognostic factors. ${ }^{26}$ A study of 2,890 cases of histologically confirmed chondrosarcomas of various locations revealed some diversity in prognosis based on histologic subtype. The following are 5-year survival rates of tumor subtypes and their encountered frequencies: chondrosarcoma, otherwise not specified (70\%); myxoid (71\%); mesenchymal (48\%); dedifferentiated (0\%); juxtacortical (93\%); chondroblastoma (85\%); clear cell (100\%). ${ }^{26}$ Currently accepted treatment paradigms and those endorsed by the authors encompass maximal but safe surgical resection with a goal of cytoreduction, followed by adjuvant fractionated radiotherapy (preferentially proton therapy). Grade 1 tumors that have been completely resected and those with minimal residual adjacent to noncritical areas can be observed.

A review of modern surgical case series of skull base chondrosarcoma revealed the use of multiple open approaches including anterior, posterolateral, transfacial, and transsphenoidal as well as endoscopic transnasal approaches. $^{27}$ Nonetheless, given the ventral origin of these tumors and their frequently extradural source and location, the authors tend to favor the endoscopic endonasal route as the primary corridor to reach the petroclival synchondrosis to remove these tumors.

\section{Malignant Meningioma}

Meningiomas are thought to be derived from the arachnoid cap cell, which is part of the cellular architecture of arachnoid granulations. These tumors are globular and encapsulated, presenting in a spectrum of areas intracranially including the convexities, parasagittal, sphenoid ridge, intraventricular, tuberculum sellae, and the infratentorial compartment involving the clivus, petroclival, and cerebellopontine angle regions; the jugular foramen; and the foramen magnum. They represent the most frequently reported primary central nervous system tumor in the United States, ${ }^{28}$ and they are considered to be benign lesions with favorable survival rates; however, on occasion they may have a high-grade subtype, associated with a poor prognosis and higher risk for metastasis. Only $3 \%$ of meningiomas are considered malignant (World Health Organization [WHO] grade III) with all subtypes, rhabdoid, papillary, and anaplastic, occurring in the petroclival area (-Fig. 5).

Formal nonbiased population-based estimates of survival of patients with meningioma were not possible until January 1,2004 , when the SEER database formally added this tumor to its records. In 2011, the first formal evaluation of this survival data revealed the most accurate data to date on the survival patterns of meningioma patients in the United States of America. According to Cahill and Claus, who looked at 12,248 meningioma patients with a 3-year follow-up, those treated with resection had an overall 3-year survival rate of 93.4\%, whereas those who did not undergo resection had a survival rate of $88.3 \% .{ }^{28}$ Young age, female sex, unilateral lesion, and surgical treatment were identified as favorable prognostic indicators. Atypical (grade II) and malignant meningiomas (grade III) had a worse prognosis. A study focusing 

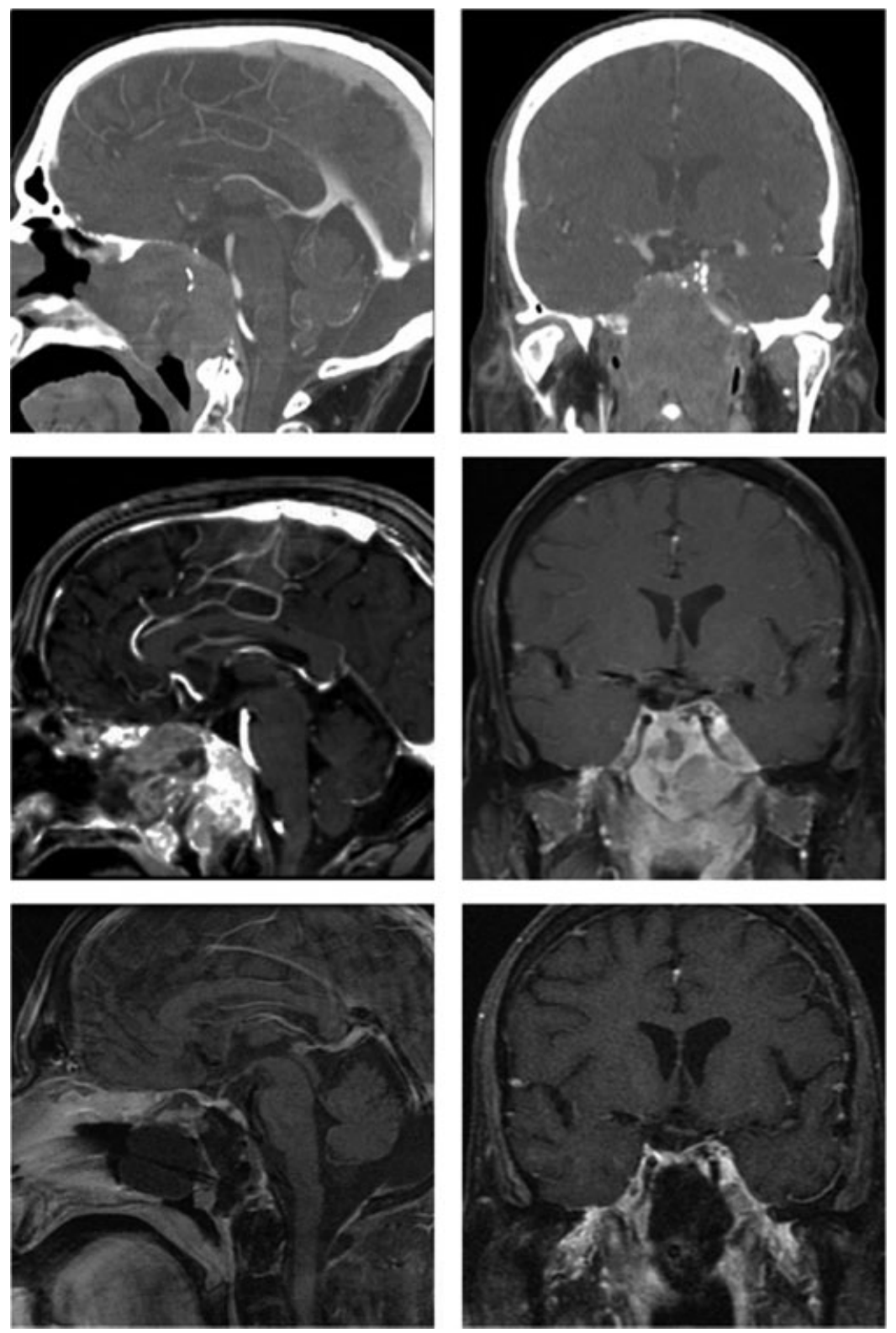

Fig. 5 A 41-year-old man, who had undergone surgery and proton beam radiotherapy for a chondrosarcoma of the clivus in his early 20 s. He presented $\sim 20$ years later with new symptoms and worsening of his double vision, pain, and a new expansile clival mass. Biopsy of the lesion revealed a spindle cell sarcoma confirmed to be a malignant meningioma (World Health Organization grade III) that was refractory to chemotherapy. An endoscopic endonasal approach for gross total resection was achieved. (Top left and right) Sagittal and axial computed tomography and arterial angiography scans demonstrating an expansile clival with mass effect on the brain stem. (Middle left and right) Preoperative magnetic resonance imaging (MRI) T1-weighted postcontrast scans demonstrating clival meningioma. (Bottom left and right) Postoperative MRI T1-weighted postcontrast scans demonstrating resection of clival meningioma and brainstem decompression.

on these more aggressive meningiomas demonstrated that in patients with atypical meningioma, the survival rate at 5 and 10 years was 95 and $79 \%$, respectively. In that same surgical series, malignant meningiomas were shown to have even lower rates at 5 and 19 years, 64.3 and $34.5 \% .^{29}$

A noncontrast CT scan shows that these lesions have either a high- or low-attenuation signal compared with surrounding brain. Hyperostosis, increased vascular markings, and calcification are characteristic findings of meningiomas on CT; however, malignant meningiomas can be more destructive of bony structures and more invasive of soft tissue, which can also be evident on MRI ( - Fig. 5). Digital subtraction angiog- raphy may be a useful study to assess the vascularity and supply of the tumor and allows the embolization of a primary feeding vessel, when present.

Similar to chordomas and chondrosarcomas, the management of malignant meningiomas in the petroclival area includes a combination of adequate surgical resection, aiming at a complete removal, followed by radiation therapy. In some cases, chemotherapy is also used. To obtain a complete resection of these tumors, the surgical team must choose a surgical corridor that facilitates the best resection of the tumor with the least morbidity. In the petroclival area, this often requires a combination of approaches. Conventionally, 
aggressive petroclival meningiomas are approached laterally through a transpetrous route combining a presigmoid and a middle fossa approach. However, although this strategy may allow the surgical team to resect a malignant petroclival meningioma in one stage, it is frequently associated to significant morbidity. ${ }^{30}$ A surgical strategy that removes infiltrated bone and dura is especially important when dealing with malignant variants. However, the surgeon must balance the goal of achieving a Simpson grade I resection against avoiding neurologic morbidity.

The combination of multiple surgical corridors around the relevant anatomy allows the surgeon to avoid dissection passing the plane of cranial nerves; therefore, optimizing the resection and decreasing morbidity. In certain situations, a single endoscopic endonasal approach can be sufficient to produce gross total resection of petroclival malignant meningiomas (-Fig. 5 ).

\section{Malignant Nerve Sheath Tumors of the Skull Base}

Malignant nerve sheath tumors (MNSTs) are rare entities that usually affect adult patients in the third to sixth decade of life, although children as young as 5 years have also harbored these tumors. ${ }^{31}$ Incidence in the overall population is $0.001 \%$ and intracranial lesions are even less common. ${ }^{31}$ Although these lesions are found in both neurofibromatosis types (NF1 and NF2), they are most often found in von Recklinghausen disease (NF1). ${ }^{31-33}$

Although there is no clear evidence regarding the etiology of MNSTs, the general consensus is that they develop de novo in patients with NF1 or that they undergo malignant transformation from a preexisting nerve sheath tumor. ${ }^{33}$ The underlying mechanism for this malignant transformation is unknown; however, biallelic loss of the tumor suppressor gene SMARCB1 may contribute. Alteration in SMARCB1 and loss of BAF47 has been found in patients harboring epithelioid MNST. ${ }^{34}$ Radiation-induced MNSTs have been diagnosed in patients who were irradiated for optic glioma, pilocytic astrocytoma, and lymphoma. ${ }^{35}$

Although theses lesions can originate from cranial nerves, this is very rare. ${ }^{33}$ When they do occur intracranially, the vestibular nerve is most often involved, followed by the facial and the trigeminal nerves. ${ }^{35}$ Due to their aggressive characteristics, any of the above can grow and erode into the petroclival region. These tumors are highly aggressive and have been associated with spinal leptomeningeal dissemination. Distant metastases have also been seen outside of the central or peripheral nervous system due to hematogenous spread.

Radiographically, MNSTs are a heterogeneous group of tumors. Noncontrast CT scan can show bone remodeling or erosion of neighboring structures; trigeminal MNSTs can invade or expand the petrous apex, foramen ovale, and foramen rotundum. ${ }^{33,36}$ Angiography is helpful in determining the location of the carotid siphon relative to the mass. Typically, the cavernous segment of the carotid artery is displaced anteromedially and inferiorly. Contrasted MRI reveals heterogeneous enhancement, irregular borders, and focal areas of necrosis; however, benign schwannomas can also have these characteristics. Perineural spread can indicate the presence of an MNST. ${ }^{37}$ - Fig. 6 demonstrates a malignant schwannoma that originated on the left trigeminal nerve with intense perineural spread.

Histopathologic analysis of these tumors reveals a high density of spindle-shaped cells arranged in a fascicular pattern. Nuclear pleomorphism and hyperchromatic cells with eosinophilic cytoplasmic granules are typical features. ${ }^{38}$ The presence of epineural invasion, perineural spread, and involvement of surrounding structures can also aid in the diagnosis. Focal areas of necrosis and mitotic figures can also be seen, lending to the malignant character of this tumor; however, the presence of benign features of a schwannoma or neurofibroma does not preclude the diagnosis of MNST. ${ }^{33}$

Nerve sheath tumors have multiple observed staining patterns. They most often stain positive for S-100 and can also stain for collagen IV. ${ }^{35}$ S-100 staining patterns can be further analyzed to help distinguish malignant lesions from benign ones: an MNST usually shows reverse expression because it is positive for $S-100 \alpha$ and negative for $S-100 \beta$ subunits. ${ }^{39}$ MNST can be differentiated from spindle cell sarcomas because of its reaction to neuron-specific enolase, desmin, and vimentin. ${ }^{33}$ Staining for p53 and $\mathrm{K}_{\mathrm{i}}-67$ antigen, which is the cellular marker for proliferation, is typically positive for malignant schwannomas.

The WHO determined three grades and four histologic subtypes for MNST. The four histologic variants include epithelioid, mesenchymal, melanotic, and glandular differentiation subtypes. ${ }^{33}$ The epithelioid variant comprises $\sim 5 \%$ of all MNST types and resembles carcinoma or amelanotic melanoma. ${ }^{40}$

Although radiosurgery is a reasonable upfront strategy for benign schwannomas, surgical extirpation of potential malignant schwannomas is warranted, particularly in cases with invasion of surrounding structures. ${ }^{41-43}$ It is critical to verify the diagnosis to a tailor a proper treatment plan and to establish reasonable expectations with the patient. Once the diagnosis is made, complete neuraxis imaging should be obtained to rule out drop metastases. Patients who have gross total resection of their lesion followed by adjuvant radiotherapy (fractionated or stereotactic) had the best survival outcomes, with the literature citing $60 \mathrm{~Gy}$ delivered in 30 fractions as an effective strategy. Chemotherapy, specifically adriamycin-based treatments, has been shown to have a limited role in treating these tumors but has been used for palliative treatment in the setting of widely metastatic disease. $^{33,40,44,45}$ Therefore, even if gross total resection is achieved, these patients should have postoperative radiation treatment. ${ }^{40,46}$

Despite gross total resection with negative surgical margins with adjuvant radiotherapy, up to half of all malignant schwannomas recur locally. ${ }^{47}$ Historically, the 5-year survival rate ranges from 37.6 to $65.7 \%{ }^{39}$ When these lesions arise in the setting of NF1, the 5-year survival rate is lower than those arising sporadically in other patients; however, there have been patients who have lived up to 7 years. ${ }^{44}$

In comparison with the previous described pathologies, surgery for MNST takes into account the inevitability of 

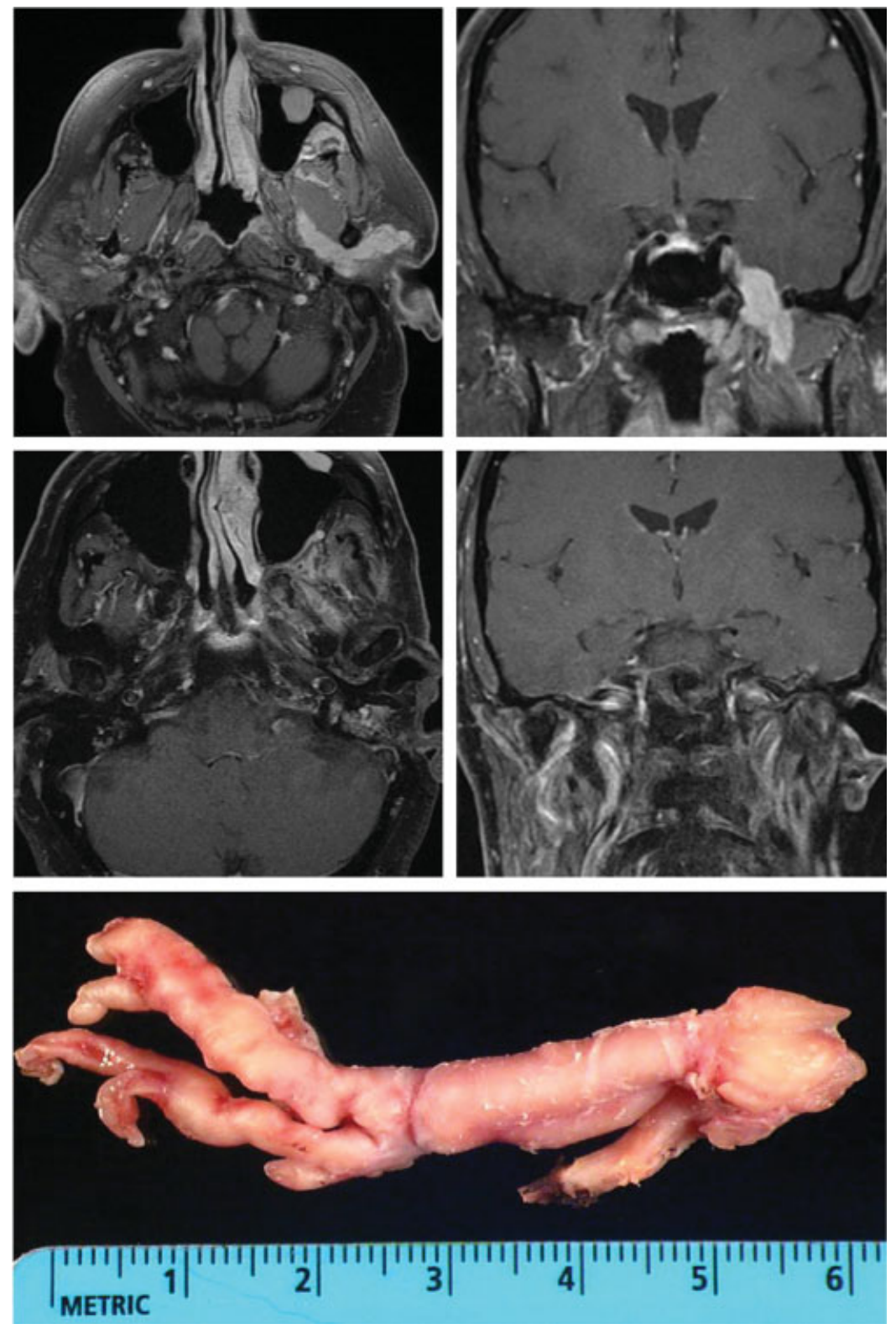

Fig. 6 Magnetic resonance imaging (MRI) and gross pathologic specimen from a patient with a malignant peripheral nerve sheath tumor. (Top left and right) Preoperative MRI T1-weighted postcontrast scans demonstrating a large extensive left-sided malignant peripheral nerve sheath tumor of the trigeminal nerve seen within the petrous bone and extending out into the parotid gland (not seen). (Middle left and right) Postoperative MRI T1-weighted postcontrast scans. (Bottom) Resection of gross specimen pictured as seen with histologic examination revealing a malignancy consistent with a malignant schwannoma.

postoperative nerve deficits. This feature can have a significant impact on surgical planning, because the nerve itself has become the lesion.

MNSTs originated from the vestibular and facial nerves are approached mainly from a retromastoid-retrosigmoid approach or translabyrinthine approach. The preservation of facial function can be a challenge in vestibular malignant schwannomas and basically impossible when they originate from facial nerves. Facial grafting or facial-hypoglossal nerve transposition should be planned accordingly in these cases. Trigeminal nerve MNST often has posterior fossa and a middle fossa component. For these cases, the surgical team must evaluate the best approach based on the anatomy and tumor relationship with the neurovascular structures. The most utilized approaches for malignant trigeminal tumors are extended infratemporal fossa; middle fossa approach and cavernous sinus peeling; retromastoid-retrosigmoid with potential suprameatal drilling to access Meckel's cave; transpetrous combining posterior fossa-middle fossa approach; endoscopic endonasal suprapetrous approach to Meckel's cave; or a combination of these approaches. -Fig. 6 shows a patient with a trigeminal malignant schwannoma that required a combination of a middle fossa approach with cavernous sinus peeling extradurally performed in association with an extended infratemporal fossa approach with removal of the mandible coronoid process. 


\section{Plasmacytoma}

Solitary plasmacytoma is a disease entity that falls in the continuum of B-cell lymphoproliferative diseases. Clinically, it is distinguished from multiple myeloma in that it is a neoplasm of a single clone of plasma cells with no other lesions found on the skeletal survey. For the diagnosis, patient bone marrow aspirates must not show evidence of myeloma, and serum and urine electrophoresis should not have the characteristic monoclonal IgG or IgA referred to collectively as M-protein. A clinical distinction between plasmacytoma and multiple myeloma is important to make because they differ in long-term prognosis. A review of 84 patients with solitary plasmacytoma and long-term follow-up demonstrated a 5year survival rate of $60 \%$ compared with the $18 \%$ rate seen with disseminated multiple myeloma at the end of 5 years. ${ }^{48}$ Ironically, multiple myeloma will develop in 55 to $60 \%$ of patients with a solitary plasmacytoma in the first 5 years and 70 to $80 \%$ by 10 years. The significantly greater survival of patients with solitary plasmacytoma underscores the prevalent use of radiotherapy as the treatment modality of choice for these tumors and makes accurate diagnosis an important distinction on initial patient presentation and future follow up.

There are ample case reports and small series in the literature with the identification of solitary plasmacytoma in the skull base particularly in the craniovertebral junction involving the occipital bone, the axis, and the atlas vertebrae. ${ }^{49,50}$ More commonly, solitary plasmacytoma involves the spine with a predilection to the pedicles. In the authors' experience, this pathology initially presents due to symptomatic compression of cranial nerves. - Fig. 7 is an example of a patient who initially presented with hearing impairment; investigation detected the presence of a large lesion in the petrous bone partially invading the infratemporal fossa and with extension around the internal carotid artery (ICA) and high cervical area. The patient was initially neurologically intact but then presented a cranial nerve VII palsy. Initial workup for multiple myeloma was negative, and a biopsy via an endoscopic endonasal approach revealed a solitary plas- macytoma to confirm the diagnosis. Two months after radiation therapy, the facial nerve deficit was resolved.

As mentioned earlier, the accepted treatment paradigm as supported by good local disease control has been biopsy and radiation treatment. Attempts at surgical resection of these entities do not increase the rates of disease control and can be complicated by excessive blood loss and cranial nerve morbidity. Monoclonal antibodies are currently used for tumors that persist after radiation therapy. However, surgery is often advocated for lesions that persist after radiation therapy, if their resection would not be associated with significant morbidity.

\section{Metastatic Disease}

Metastatic disease to the petroclival region is rare and will be discussed briefly. The few cases in the literature indicate that there are no epidemiologic or demographic patterns that can be reported with any reliability. Usually when metastatic disease is found in the petroclival area, patients present with a mononeuropathy of cranial nerves III, IV, and VI or the trigeminal nerve. ${ }^{51-53}$ Although mass lesions have been found in the skull base as explanations for this clinical presentation, patients who have a cancer diagnosis could also harbor carcinomatosis. - Fig. 8 demonstrates the case of a 61-year-old man who presented with right cranial nerve VI palsy and a clival lesion as demonstrated on MRI. Although the lesion was dark in T2, the possible diagnosis of a chordoma or another primary bony lesion was considered. He had no previous diagnosis, and an endoscopic endonasal approach was used for resection of the tumor. The tumor was completely removed, and pathologic exam revealed a thyroid carcinoma. The patient underwent resection of the primary tumor, with the specimen depicted in - Fig. 8, followed by systemic treatment.

Follicular carcinoma of the thyroid gland and breast carcinoma are common examples of the type of metastases that have been found in the petroclival region. ${ }^{51,54}$ The case examples of the metastatic disease to this location are often approached surgically with the goals of biopsy and
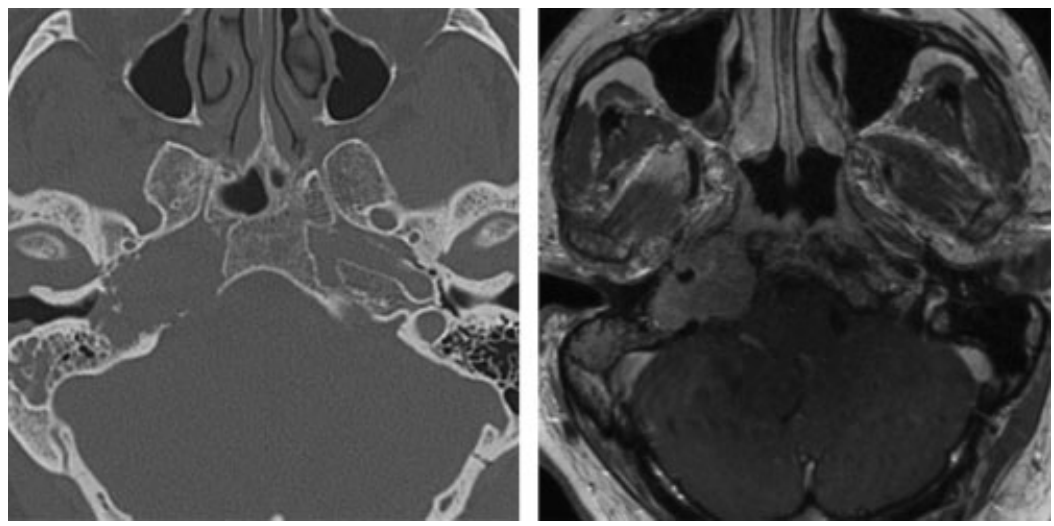

Fig. 7 A 51-year-old man presented initially with some right-sided hearing symptoms, and investigation showed the presence of a large destructive lesion involving the right petrous apex and internal auditory canal with extension anterior to the skull base, the right internal auditory canal, on computed tomography scans (left). Magnetic resonance postcontrast scans (right) confirm an enhancing lesion in the petrous bone with intracranial extension into the right cerebellopontine angle cistern with extension around the internal carotid artery and high cervical area. Days later in the middle of his medical workup that was largely negative for multiple myeloma, he presented with a seventh cranial nerve palsy. An endoscopic endonasal approach confirmed the pathology was consistent with a plasmacytoma. 

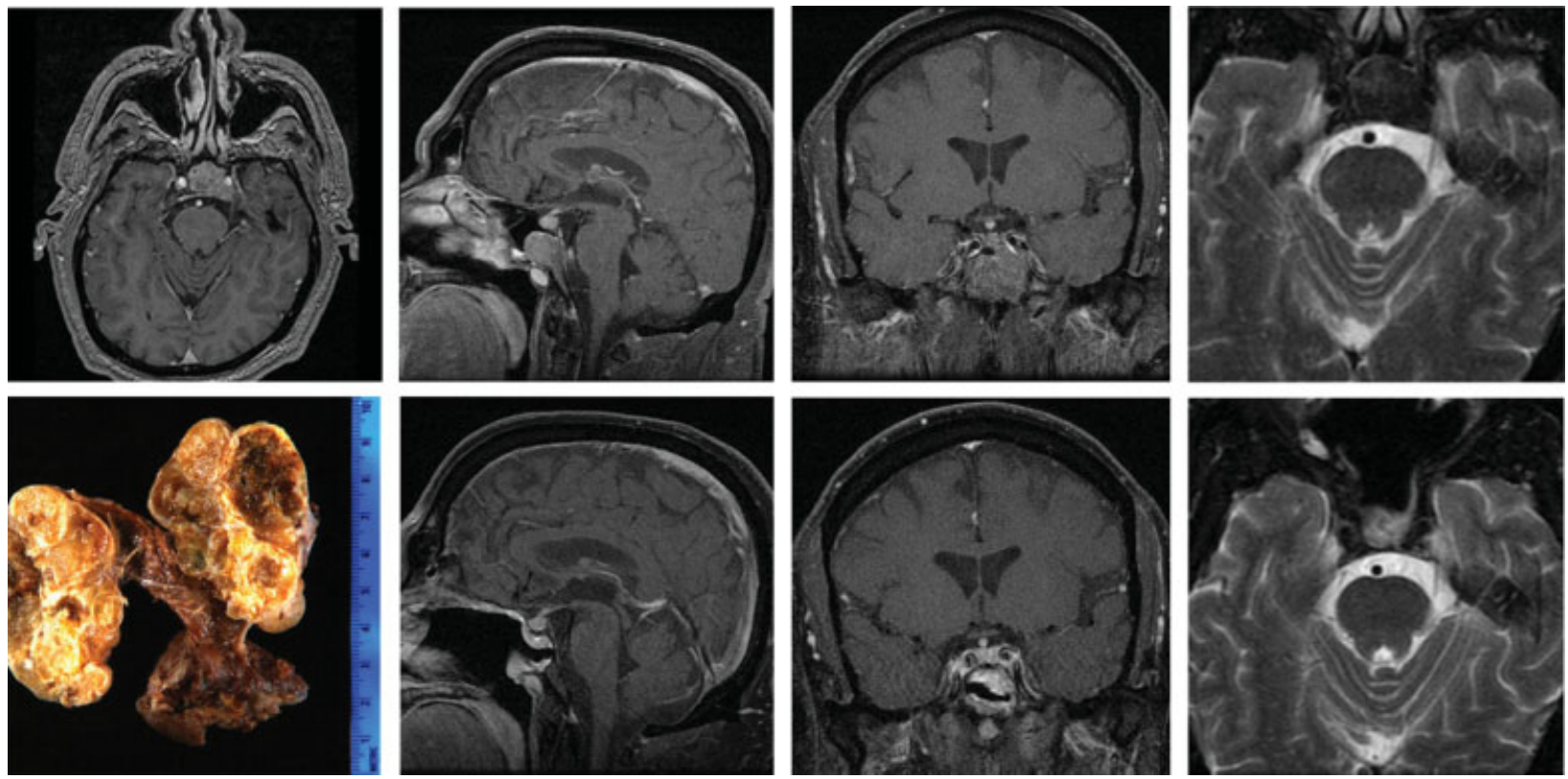

Fig. 8 A 61-year-old patient presented with cranial nerve VI palsy and a midline clival mass, possibly chordoma. (Top left to right) Preoperative magnetic resonance imaging (MRI) T1-weighted postcontrast scans demonstrating a focal midline moderately enhancing clival mass. T2weighted sequences revealed a hypointense mass atypical for chordoma (top row, fourth panel on the right). Endoscopic endonasal approach and resection of mass revealed a metastatic thyroid carcinoma. (Bottom left and right) Postoperative MRI T1-weighted postcontrast scans. Bottom row also includes gross specimen pictured as seen with histologic examination revealing a malignancy consistent with thyroid carcinoma.

decompression followed by radiation therapy. The goal of surgery in patients with petroclival metastases is rarely that of complete resection and often is necessary for diagnostic confirmation helping to define the treatment strategy as well as palliation. A systemic evaluation is always necessary when there is high possibility for the lesion to be metastatic to define the primary cancer and patient's systemic condition and tumor burden. It is not prudent to subject a patient with advanced disease (i.e., multiple synchronous metastatic lesions) to complex morbid procedures that will not help or control already disseminated systemic disease. Generally, as with other metastatic processes to the brain, metastases to the skull base portend a poor prognosis. ${ }^{52}$ Various strategies may be appropriate for the management of petroclival metastasis, ranging from no treatment, as what is commonly encountered in patients in whom the primary tumor is known and have advanced systemic disease, to aggressive resections of solitary metastasis.

If current imaging techniques reveal a lesion that cannot explain the patient's symptoms, the patient should undergo serial lumbar punctures for cerebrospinal fluid cytologic analysis. Discussion between a team including neurosurgeons, medical oncologists, and radiation oncologist should ensue. As with any other malignancies, the patient should then be educated regarding the goals of care and reasonable, expected outcomes from the treatment paradigm. In patients with a solitary lesion with a known biology that is resistant to radiotherapy, surgical excision followed by adjuvant radiation with or without chemotherapy should be entertained. Depending on the overall medical condition of the patient, either palliative radiation with or without chemotherapy or hospice may be the most appropriate option.
The treatment plan with regards to systemic metastases to the cranial vault is complex due to the multiple variables that need to be considered; a tumor board consisting of multiple experts from different medical and surgical fields is necessary to tailor the best treatment for each individual patient.

\section{Discussion}

\section{Surgical Planning and Decision Making}

As a multidisciplinary team performs the global management of malignant petroclival tumors, it is important that all those involved in the process define the strategic plan of treatment promptly. All skull base cases should be discussed in conference until a consensus on surgical approach and overall management is reached among radiologists, neuro-oncologists, neurosurgeons, otolaryngologists, head and neck surgeons, and radiation oncologists. The initial decision must be contextualized in terms of treatment goals and the need for any surgical intervention. As previously described, some patients with metastatic tumors to the skull base may have disseminated systemic disease and the initial assessment may be to define the need for palliative procedures. In other instances, there may be a need for diagnosis to define the best line of care without a need for tumor resection. Examples of petroclival malignances in this situation are lymphomas, plasmacytomas, and metastatic tumors. Once surgical intervention has been identified as the best primary treatment, the team should collectively decide on the approach that will allow maximal tumor removal with minimal need for cranial nerve or neural tissue displacement or retraction while avoiding the risk for vascular compromise. With this in mind, patients can be offered an endonasal or a transcranial 
approach or a combination of both when complex, multicompartment tumors present (-Fig. 9).

There are multiple approaches at the disposal of the skull base surgeon that can be used when accessing lesions involving the petroclival region. Anteromedial (endonasal route), anterolateral (orbitozygomatic approach and anterior petrosectomy), lateral (transpetrous presigmoid-posterior petrosectomy), and posterior (retromastoid-retrosigmoid approach) trajectories have been formulated to reach this area while respecting the complex local neurovascular structures; each of these approaches has its limitations and advantages.

Generally speaking, an endoscopic endonasal approach is an anteromedial approach that is ideal for median lesions involving the clivus, medial to the ICA. Some tumors with lateral extensions can still be approached primarily using a ventral approach as long as the tumor has created a corridor pushing the important neurovascular structures to the periphery. Chondrosarcomas exemplify a tumor that can become extremely complex starting in the petroclival synchondrosis and extending into multiple areas to involve the middle cranial fossa, posterior cranial fossa, and even the upper portion of the neck, which can still be completely resected through an expanded endonasal approach. In these cases, often there is a need for resection of the eustachian tube to allow for complete tumor exposure and resection. Even lateral tumor extension posterior to the paraclival internal carotid arteries can be approached endonasally, and this often requires the removal of the bony carotid canals to allow ICA lateralization and better reach posterolaterally. On the other hand, if there is tumor that extends lateral to any of the cranial nerves, they are better approached from a lateral route. Ultimately, the approach that is chosen depends on how the tumor engulfs or displaces cranial nerves, the carotid, and the basilar arteries.

The majority of petroclival malignant tumors can be addressed via endoscopic endonasal approach either obtaining a gross total resection or aiming at a subtotal excision as part of a multicorridor strategy. When tumors present invasion of multiple cranial compartments (cavernous sinus, middle fossa, posterior fossa), going beyond the plane of cranial nerves, the endoscopic endonasal approach must be supplemented with open approaches during the same anesthesia or as a second stage after the patient's recovery. Furthermore, if the petroclival tumor seems to be suitable for total resection through an open approach in a single stage, then that should also be taken in consideration and the less morbid alternative should be chosen by the multidisciplinary team.

\section{Open Surgical Approaches}

The Transpetrosal Approach (Variants: Presigmoid, Posterior Petrosectomy, Retrolabyrinthine, Transcrusal, Transcochlear)

This approach is used to access lesions that compromise the middle and upper third of petroclival area that mainly involve
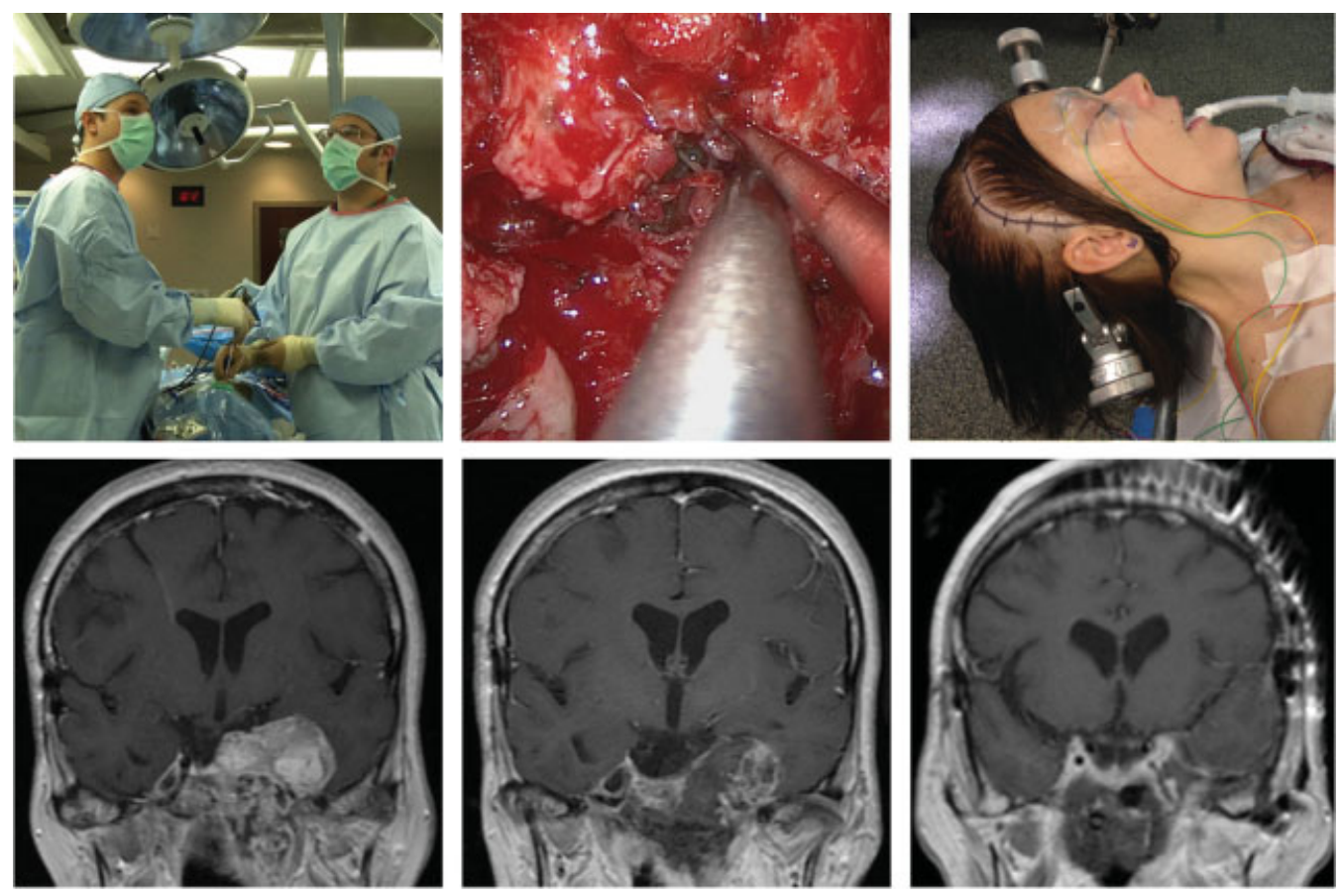

Fig. 9 Our surgical setup includes preparations for endoscopic endonasal approaches (EEAs), open craniotomies, and combined approaches to pathologies of the skull base. (Top left and middle) Our endoscopic endonasal approach surgical suite with two operating teams, neurosurgery and otolaryngology, positioned on either side of the patient with dual video monitors in their field of view. (Middle) A screen shot of an operator's field of view on the monitor during dissection. (Top right) An open craniotomy after the completion of a first-stage endoscopic endonasal approach. (Bottom) A skull base chordoma in a 47-year-old woman with lateral extension that required a two-stage procedure. (Left) Preoperative magnetic resonance imaging (MRI) T1-weighted postcontrast scans demonstrating the large chordoma with lateral extension. (Middle) Postoperative MRI after an EEA with residual lateral tumor that could not be reached. (Right) Postoperative MRI after open pterional craniotomy and gross total resection of enhancing remnant tumor. 
the petrous bone. ${ }^{55}$ The craniectomy begins with a full mastoidectomy so that the middle fossa and posterior fossa dura are exposed; this exposure allows visualization of the transverse-sigmoid junction and the exposure of the superior petrosal sinus. There are variants of this approach, and the surgeon has the option of performing a complete translabyrinthine approach by drilling the semicircular canals or preserving them by performing a presigmoid retrolabyrinthine approach when hearing preservation is desired. The facial nerve canal is identified inside the mastoid in the most anterior compartment and should be left inside the cortical bone in cases there is no need for facial nerve transposition. The internal acoustic canal can be exposed using this approach, and extension of the lesion at that level can be addressed. This approach can also be extended to the middle fossa with dural opening and elevation of the temporal lobe. Attention should be given to the vein of Labbé when this maneuver is performed to avoid a disastrous temporal venous infarct. At this point, the temporal lobe is retracted and the fourth cranial nerve identified in relation to the tentorium; the superior petrosal sinus and the tentorium are then divided to expose the posterior fossa and middle fossa in direct relationship with the petroclival region. For more invasive lesions in which hearing is already compromised, medial drilling inferior to the internal acoustic canal as well as resection of the cochlea can achieve further anteromedial exposure. With these extensions, the clivus can be exposed medially as well as the ICA anteriorly. The cranial nerve VI runs just above the petrous apex lateral to the internal auditory canal (IAC) as it runs under the Gruber ligament at the Dorello canal; thus, care should be taken at that level during the very deep drilling maneuvers.

Moreover, there are four successive stages when approaching the petroclival region through a petrosectomy. ${ }^{55}$ The retrolabyrinthine stage exposes the three semicircular canals. As more of the petrous apex is drilled medially in the transcrusal stage, the superior and inferior semicircular canals are removed, allowing for more access toward the clivus and allowing for hearing preservation. The transotic portion of the exposure involves the complete removal of all semicircular canals and skeletonization of the facial nerve. The transcochlear variation involves sectioning the greater superficial petrosal nerve, mobilizing the facial nerve posteriorly, removing the cochlea and exposing the petrous ICA. Based on cadaveric studies, the transcrusal approach offers the largest corridor to the petroclival area while limiting cranial neuropathy and unnecessary risk to the ICA. ${ }^{55}$ However, one needs to understand that once a retrolabyrinthine or a transcrusal transpetrous approach is completed, the angle of view of the clivus and contralateral sixth cranial nerve is optimized, but the sight of the prepontine cistern is still partially blocked by the cranial nerves VII and VIII in the same proportion of a simple retrosigmoid approach. The surgeon can only have a full visualization anterior to cranial nerves VII and VIII in the cistern if a complete transcochlear approach is performed with mobilization of the seventh cranial nerve (i.e., potential for morbidity). It is for that reason that the transpetrous approaches are progressively less utilized. However, they remain a great option for bony lesions lateral to the IAC.

\section{Anterior Transpetrosal Approach ("Shiobara-Kawase Approach")}

This approach offers an optimal extradural view of the clival region while exposing the trigeminal nerve and the seven and eight cranial nerve complex. ${ }^{56}$ Because this is an entirely extradural approach, excessive retraction on the temporal lobe is reduced. The craniotomy is $\sim 4 \times 4 \mathrm{~cm}$ with two-thirds of it anterior to the external auditory canal and one-third posterior to it; the most inferior margin of the bone flap is level with the middle fossa floor. ${ }^{56}$ In this extradural approach, the dura of the temporal lobe is lifted to expose the floor of the middle fossa. The arcuate eminence is a key landmark in the floor of the middle cranial fossa that defines the lateral limit of the approach. To reach this point, which identifies the superior semicircular canals, the middle meningeal artery must be transected to allow for greater elevation of the dura off the floor. The third branch of the trigeminal nerve is then encountered entering the foramen ovale. As the dura is lifted, the arcuate eminence can be used to find the greater superficial petrosal nerve anteriorly and the porus trigeminus medially. The greater superficial petrosal nerve is gently dissected and the dura is lifted further until the gasserian ganglion is visualized. The anterior limit of the approach is the mandibular branch of the trigeminal nerve and the medial posterior limit is the petrous ridge.

Once these structures are identified, the quadrangular bone in between them is drilled to unroof the IAC, which ultimately allows for exposure of the intracanalicular dura, the posterior fossa dura, as well as the superior and inferior petrosal sinuses. It is critical to be aware of the close proximity of the cochlea to the genu of the petrous carotid artery during this exposure; the cochlea lies within the lateral half of the postmeatal triangle, which is a lateral wedge of bone between the IAC and the superior semicircular canal. ${ }^{56}$ In addition, the lateral aspect of the Dorello canal and the undersurface of the Meckel cave are exposed, all of which is done in an extradural fashion. This approach allows for a craniectomy extending from the arcuate eminence to the petrous apex and exposes the IAC and the clivus while avoiding cranial nerve and vascular transposition; however, it is limited because it is difficult to access lesions that extend into the cavernous sinus, behind the sigmoid sinus, or below the zygoma. ${ }^{56}$ On the other hand, it can be expanded by opening the dura of the Meckel cave and/or the dura of the posterior fossa, allowing a complete exposure of the cistern between the cranial nerves VI and VII in the ipsilateral side as well full visualization of the trigeminal nerve from its origin to its trifurcation.

\section{Retrosigmoid Approach}

The retrosigmoid approach (also known as retromastoid or lateral suboccipital approach) is the most commonly employed technique to access the cerebellopontine angle and petroclival regions. Some technical variations regarding 
incision length and orientation, patient positioning (lateral versus supine versus semisitting position), as well as craniotomy versus craniectomy have been described. In brief, bone removal angled on the asterion flanks the transverse and sigmoid sinuses and their junction; the dura is opened in arch fashion and reflected outward, covering the exposed sinuses. The authors favor a small initial opening on the lower aspect of the dural exposure to grant lateral access to the medullary cistern. This enables early cerebrospinal fluid drainage and cerebellar relaxation. This simple yet versatile approach enables visualization of the entire lateral aspect of the posterior fossa, from the tentorium to foramen magnum. ${ }^{57}$ Cranial nerves III through XI are readily accessible, as well as the vertebral, anterior inferior cerebellar, and posterior inferior cerebellar arteries and their terminal branches. Combining the initial exposure with intradural drill of the suprameatal region also grants access to the middle fossa. ${ }^{58}$

Typically utilized in the treatment of neurovascular conflicts (especially trigeminal neuralgia and hemifacial spasm) and resection of vestibular schwannomas, the retrosigmoid approach has gained renewed popularity over the last decade. Whether in conjunction with other approaches ${ }^{59}$ or on its own, ${ }^{60}$ this technique is effective in the treatment of complex tumors of the petroclival region; some authors have advocated its use in the place of more complex approaches. ${ }^{61}$ Although the authors agree with this rationale in regards to benign petroclival tumors, the case is not the same when dealing with malignancies of the region, especially the two most common pathologies, chordomas and chondrosarcomas. As previously stated, these tumors typically rise from the clivus and the petroclival synchondrosis; their growth patterns imply displacement of the dura and neurovascular contents posteriorly. Approaching them through a posterior-lateral trajectory (i.e., retrosigmoid approach), although enticing at first given the familiarity of most surgeons with the technique and its inherent simplicity, implies traversing an often disease-free intradural corridor to reach the target. It is the authors' experience that, in a significant number of instances and particularly regarding chondrosarcomas, these tumors have merely displaced the petroclival dura or have minimally violated its integrity. This finding is of paramount significance when selecting which surgical corridor must be employed; the endonasal approach not only enables direct ventral access to the region without the need for dural transgression but also provides an angle for tumor resection free of cranial nerve manipulation, given that these have been pushed posteriorly by the tumor. Furthermore, the endonasal corridor also permits simultaneous access to the most ventral aspect of the posterior fossa as well as to the contralateral petroclival region, a feature most valuable when dealing with complex, multicompartment tumors, as often seen in chordomas. Finally, when the dura has not been violated by the tumor, the endonasal approach potentially avoids intradural seeding, a feared complication of chordomas. For these reasons, the authors favor endoscopic endonasal approaches over the retrosigmoid approach for the majority of petroclival malignancies.

\section{Endoscopic Approaches}

Endoscopic endonasal approaches to the petroclival region have replaced all ventral open approaches (facial degloving, Le Fort osteotomies, transfacial and transbasal approaches, etc.) allowing the same or better exposure with less morbidity for the patients. An endoscopic endonasal approach to the petroclival area requires a transpterygoid approach. ${ }^{62,63}$ Because of the risk of neurovascular injury with these expanded cases and because of the need for electrophysiologic monitoring, the patient is secured in a Mayfield three-pin head holder with the head turned to the right (in case of a right-handed surgeon), translated anteriorly. Relevant cranial nerves and sensory evoked potentials are monitored during the surgery. Because the authors favor a four-handed, twosurgeon technique, two monitors are positioned across from the surgeons. The surgeons stand parallel to each other on the right side of the patient if both physicians are right-hand dominant or with one surgeon on each side of the patient when one of the surgeons is left-handed (-Fig. 9). Neuronavigation is also utilized during the procedure, and the system is placed at the head of the bed.

A transpterygoid approach begins with a complete dissection of the medial sinonasal corridor to access the sphenoid sinus. Prior to beginning the transpterygoid corridor, a pedicled nasoseptal flap is harvested from the contralateral side due to the high risk of cerebrospinal fluid leak and to cover a denuded ICA at the end of the procedure. Subsequent to preparing the neurovascular flap, a "reverse" flap is harvested and immediately used to cover the donor site of the nasoseptal flap.

To access the sphenoid sinus the middle turbinate is first removed. Its mucosa can be used at the conclusion of the case for reconstruction. The inferior half of the superior turbinate is removed to allow for visualization of the natural ostium of the sphenoid sinus, which is then widened medially using Kerrison rongeurs. The roof and the lateral wall of the sphenoid sinus are visualized and the sphenoidotomy is widened bilaterally, which is essential for allowing sufficient space for the instruments, satisfactory freedom of movement, and proper visualization of anatomical landmarks. Once the posterior nasal septum has been detached from the rostrum, the basopharyngeal fascia can then be dissected from the inferior floor of the sphenoid sinus and the medial pterygoid plate. The floor is then drilled toward the clival recess and laterally in the direction of the foramen lacerum. Next, the sphenopalatine foramen (or foramina) and its associated arteries are exposed. At this point, the sphenopalatine artery and posterior nasal artery are isolated and cauterized with bipolar electrocautery. The vidian neurovascular bundle is transected, and the soft tissue contents of the pterygopalatine fossa are lateralized. This maneuver allows the posterior dissection of the vidian canal in the direction of the lacerum segment of the ICA. The level of the vidian nerve marks the same level of the petrous ICA, which is helpful information while dealing with malignant tumor that surrounds the vessel.

The medial pterygoid plate is isolated and drilled in a caudal-to-rostral orientation to avoid injury to the horizontal 
segment of the petrous ICA. A coarse diamond 3-mm bur is used to drill the medial pterygoid plate toward the middle cranial fossa. Drilling can proceed circumferentially around the vidian canal to reach the foramen lacerum; here, the vidian artery will be visualized as a branch from the anterior genu of the petrous ICA. The vidian canal can be identified by its position, which is immediately lateral to the junction of the sphenoid sinus floor and the medial pterygoid plate. The medial portion of the clivus must also be drilled to assess the petroclival region. If needed, further lateral dissection continues to drill the lateral pterygoid plate in a caudal-torostral fashion until it is also flush with the middle cranial fossa and foramen ovale. This basic transpterygoid corridor can be extended or curtailed according to the needs of the surgery.

Once the ICA is completely identified with its petrous, lacerum, and paraclival segments, the drilling proceeds on the middle cranial fossa floor as well as around the ICA canal. Once this bone is thin, it is elevated from the middle cranial fossa dura, which is completely exposed. The clivus is drilled posteriorly until the dura is also exposed at that level. There will be remains of bone medial and posterior to the clival ICA bilateral. It is very important to consistently expose the ICA in these approaches to allow lateral retraction. With this maneuver, the retro-ICA space and petrous apex are reached, and further lateral drilling can be performed using angled drills.

As petroclival malignant tumors often spread into the occipital condyle, jugular foramen, and upper cervical area, it is essential to remove the eustachian tube to create a direct corridor into the most inferior aspect of the inferior petrosal sinus and petroclival synchondrosis. The eustachian tube must be removed carefully because the lateral cuts are performed in intimate proximity with the parapharyngeal ICA. Once the eustachian tube is removed, the petrous bone is exposed and it can be drilled below the level of the petrous ICA. As this maneuver proceeds laterally and inferiorly, it follows the petroclival synchondrosis at the level of the jugular foramen and lesion in that region can be explored and resected. The occipital condyle can also be drilled depending on the extension of the tumor.

Posteriorly, petroclival malignances can insinuate or invade the dura reaching the cerebellopontine angle or the cerebellomedullary cistern. Frequently, the tumor can be followed safely into those areas. When dealing with chordomas and chondrosarcomas, the surgeon often encounter a layer of dura that still is preserved, preventing the tumor from reaching the subarachnoid space.

\section{Final Comments}

When approaching a patient with a petroclival region mass, one should first focus on the goals of treatment and the need for surgical intervention. Very often, a diagnosis needs to be established while limiting morbidity and maintaining the patient's neurologic status and quality of life. The treatment plan should then be tailored according to the natural history of the disease and to the individual patient comorbidities. If surgery is indicated, a patient may need a single- or multiple-stage procedure to achieve a gross total resection while minimizing neurologic deficit. Frequently, adjuvant therapies are indicated. Although chemotherapy may have a role in select cases, radiotherapy is a consistent part of the multimodal treatment paradigm. A concerted effort of physicians from multiple specialties is crucial to ensure the best possible outcomes.

\section{References}

1 Healey JH, Lane JM. Chordoma: a critical review of diagnosis and treatment. Orthop Clin North Am 1989;20(3):417-426

2 McMaster ML, Goldstein AM, Bromley CM, Ishibe N, Parry DM. Chordoma: incidence and survival patterns in the United States, 1973-1995. Cancer Causes Control 2001;12(1):1-11

3 Yang XR, Ng D, Alcorta DA, et al. T (brachyury) gene duplication confers major susceptibility to familial chordoma. Nat Genet 2009; 41(11):1176-1178

4 Vujovic S, Henderson S, Presneau N, et al. Brachyury, a crucial regulator of notochordal development, is a novel biomarker for chordomas. J Pathol 2006;209(2):157-165

5 Henderson SR, Guiliano D, Presneau N, et al. A molecular map of mesenchymal tumors. Genome Biol 2005;6(9):R76

6 Fernando RI, Litzinger M, Trono P, Hamilton DH, Schlom J, Palena C. The T-box transcription factor Brachyury promotes epithelialmesenchymal transition in human tumor cells. J Clin Invest 2010;120(2):533-544

7 Bergh P, Kindblom LG, Gunterberg B, Remotti F, Ryd W, MeisKindblom JM. Prognostic factors in chordoma of the sacrum and mobile spine: a study of 39 patients. Cancer 2000;88(9): 2122-2134

8 Schwab JH, Boland PJ, Agaram NP, et al. Chordoma and chondrosarcoma gene profile: implications for immunotherapy. Cancer Immunol Immunother 2009;58(3):339-349

9 Wold LE, Laws ER Jr. Cranial chordomas in children and young adults. J Neurosurg 1983;59(6):1043-1047

10 Jahangiri A, Jian B, Miller L, El-Sayed IH, Aghi MK. Skull base chordomas: clinical features, prognostic factors, and therapeutics. Neurosurg Clin N Am 2013;24(1):79-88

11 Llauger J, Palmer J, Amores S, Bagué S, Camins A. Primary tumors of the sacrum: diagnostic imaging. AJR Am J Roentgenol 2000; 174(2):417-424

12 Rossleigh MA, Smith J, Yeh SD. Scintigraphic features of primary sacral tumors. J Nucl Med 1986;27(5):627-630

13 Boriani S, Chevalley F, Weinstein JN, et al. Chordoma of the spine above the sacrum. Treatment and outcome in 21 cases. Spine 1996; 21(13):1569-1577

14 Suit HD, Goitein M, Munzenrider J, et al. Definitive radiation therapy for chordoma and chondrosarcoma of base of skull and cervical spine. J Neurosurg 1982;56(3):377-385

15 Austin-Seymour M, Munzenrider JE, Goitein M, et al. Progress in low-LET heavy particle therapy: intracranial and paracranial tumors and uveal melanomas. Radiat Res Suppl 1985;8: S219-S226

16 Austin-Seymour M, Munzenrider J, Linggood R, et al. Fractionated proton radiation therapy of cranial and intracranial tumors. Am J Clin Oncol 1990;13(4):327-330

17 Richardson MS. Pathology of skull base tumors. Otolaryngol Clin North Am 2001;34(6):1025-1042, vii

18 Yen $\mathrm{CH}$, Chang CY, Teng MM, et al. Different and identical features of chondroblastic osteosarcoma and chondrosarcoma: highlights on radiography and magnetic resonance imaging. J Chin Med Assoc 2009;72(2):76-82 
19 Crapanzano JP, Ali SZ, Ginsberg MS, Zakowski MF. Chordoma: a cytologic study with histologic and radiologic correlation. Cancer 2001;93(1):40-51

20 Mitchell A, Scheithauer BW, Unni KK, Forsyth PJ, Wold LE, McGivney DJ. Chordoma and chondroid neoplasms of the spheno-occiput. An immunohistochemical study of 41 cases with prognostic and nosologic implications. Cancer 1993;72(10):2943-2949

21 Abenoza P, Sibley RK. Chordoma: an immunohistologic study. Hum Pathol 1986;17(7):744-747

22 Oakley GJ, Fuhrer K, Seethala RR. Brachyury, SOX-9, and podoplanin, new markers in the skull base chordoma vs chondrosarcoma differential: a tissue microarray-based comparative analysis. Mod Pathol 2008;21(12):1461-1469

23 Sangoi AR, Karamchandani J, Lane B, et al. Specificity of brachyury in the distinction of chordoma from clear cell renal cell carcinoma and germ cell tumors: a study of 305 cases. Mod Pathol 2011; 24(3):425-429

24 Shen J, Li CD, Yang HL, et al. Classic chordoma coexisting with benign notochordal cell rest demonstrating different immunohistological expression patterns of brachyury and galectin-3. J Clin Neurosci 2011;18(1):96-99

25 Oghalai JS, Buxbaum JL, Jackler RK, McDermott MW. Skull base chondrosarcoma originating from the petroclival junction. Otol Neurotol 2005;26(5):1052-1060

26 Giuffrida AY, Burgueno JE, Koniaris LG, Gutierrez JC, Duncan R, Scully SP. Chondrosarcoma in the United States (1973 to 2003): an analysis of 2890 cases from the SEER database. J Bone Joint Surg Am 2009;91(5):1063-1072

27 Bloch O, Parsa AT. Skull base chondrosarcoma: evidence-based treatment paradigms. Neurosurg Clin N Am 2013;24(1):89-96

28 Cahill KS, Claus EB. Treatment and survival of patients with nonmalignant intracranial meningioma: results from the Surveillance, Epidemiology, and End Results Program of the National Cancer Institute. Clinical article. J Neurosurg 2011;115(2): 259-267

29 Palma L, Celli P, Franco C, Cervoni L, Cantore G. Long-term prognosis for atypical and malignant meningiomas: a study of 71 surgical cases. J Neurosurg 1997;86(5):793-800

30 Almefty R, Dunn IF, Pravdenkova S, Abolfotoh M, Al-Mefty O. True petroclival meningiomas: results of surgical management. J Neurosurg 2014;120(1):40-51

31 Nakayama Y, Watanabe M, Suzuki K, et al. Malignant peripheral nerve sheath tumor of the trigeminal nerve: clinicopathologic features in a young adult patient. Neuropathology 2013;33(5): 541-546

32 Ducatman BS, Scheithauer BW, Piepgras DG, Reiman HM, Ilstrup DM. Malignant peripheral nerve sheath tumors. A clinicopathologic study of 120 cases. Cancer 1986;57(10):2006-2021

33 Ziadi A, Saliba I. Malignant peripheral nerve sheath tumor of intracranial nerve: a case series review. Auris Nasus Larynx 2010;37(5):539-545

34 Rizzo D, Fréneaux P, Brisse H, et al. SMARCB1 deficiency in tumors from the peripheral nervous system: a link between schwannomas and rhabdoid tumors? Am J Surg Pathol 2012;36(7):964-972

35 Scheithauer BW, Erdogan S, Rodriguez FJ, et al. Malignant peripheral nerve sheath tumors of cranial nerves and intracranial contents: a clinicopathologic study of 17 cases. Am J Surg Pathol 2009; 33(3):325-338

36 Hedeman LS, Lewinsky BS, Lochridge GK, Trevor R. Primary malignant schwannoma of the Gasserian ganglion. Report of two cases. J Neurosurg 1978;48(2):279-283

37 Parker GD, Harnsberger HR. Clinical-radiologic issues in perineural tumor spread of malignant diseases of the extracranial head and neck. Radiographics 1991;11(3):383-399

38 Akimoto J, Ito H, Kudo M. Primary intracranial malignant schwannoma of trigeminal nerve. A case report with review of the literature. Acta Neurochir (Wien) 2000;142(5):591-595
39 Stone JA, Cooper H, Castillo M, Mukherji SK. Malignant schwannoma of the trigeminal nerve. AJNR Am J Neuroradiol 2001;22(3): 505-507

40 Fisher BJ, Dennis KE. Malignant epithelioid cranial nerve sheath tumor: case report of a radiation response. J Neurooncol 2006; 78(2):173-177

41 Pollock BE, Foote RL, Stafford SL. Stereotactic radiosurgery: the preferred management for patients with nonvestibular schwannomas? Int J Radiat Oncol Biol Phys 2002;52(4):1002-1007

42 Day JD, Fukushima T. The surgical management of trigeminal neuromas. Neurosurgery 1998;42(2):233-240, discussion 240241

43 McCormick PC, Bello JA, Post KD. Trigeminal schwannoma. Surgical series of 14 cases with review of the literature. J Neurosurg 1988;69(6):850-860

44 Rossiter JP, Fenton PV. Malignant peripheral nerve sheath tumor in neurofibromatosis type 1. JAMA Neurol 2014;71(2):242

45 Sordillo PP, Helson L, Hajdu SI, et al. Malignant schwannomaclinical characteristics, survival, and response to therapy. Cancer 1981;47(10):2503-2509

46 Ganapathy K, Ghosh M. Clavicular metastasis from malignant trigeminal schwannoma. J Clin Neurosci 2003;10(4):485-492

47 Bailet JW, Abemayor E, Andrews JC, Rowland JP, Fu YS, Dawson DE. Malignant nerve sheath tumors of the head and neck: a combined experience from two university hospitals. Laryngoscope 1991; 101(10):1044-1049

48 McLain RF, Weinstein JN. Solitary plasmacytomas of the spine: a review of 84 cases. J Spinal Disord 1989;2(2):69-74

49 Piper JG, Menezes AH. Management strategies for tumors of the axis vertebra. J Neurosurg 1996;84(4):543-551

50 Ahn AI, Wren MK, Meyer TA. Skull base plasmacytoma with conductive hearing loss and an external auditory canal mass. Ear Nose Throat J 2012;91(7):E1-E5

51 Hirota N, Fujimoto T, Takahashi M, Fukushima Y. Isolated trigeminal nerve metastases from breast cancer: an unusual cause of trigeminal mononeuropathy. Surg Neurol 1998;49(5): 558-561

52 Rubinstein MK. Cranial mononeuropathy as the first sign of intracranial metastases. Ann Intern Med 1969;70(1):49-54

53 Barron KD, Rowland LP, Zimmerman HM. Neuropathy with malignant tumor metastases. J Nerv Ment Dis 1960;131:10-31

54 Ehrmann J, Dusková M, Machac J, Benýsek V, Hobza J, Kolár Z. Solitary intracranial metastasis of follicular carcinoma of the thyroid gland clinically mimicking a meningioma. Cesk Patol 2004;40(2):68-71

55 Horgan MA, Anderson GJ, Kellogg JX, et al. Classification and quantification of the petrosal approach to the petroclival region. J Neurosurg 2000;93(1):108-112

56 Day JD, Fukushima T, Giannotta SL. Microanatomical study of the extradural middle fossa approach to the petroclival and posterior cavernous sinus region: description of the rhomboid construct. Neurosurgery 1994;34(6):1009-1016, discussion 1016

57 Rhoton AL Jr. The cerebellopontine angle and posterior fossa cranial nerves by the retrosigmoid approach. Neurosurgery 2000;47(3, Suppl):S93-S129

58 Samii M, Tatagiba M, Carvalho GA. Retrosigmoid intradural suprameatal approach to Meckel's cave and the middle fossa: surgical technique and outcome. J Neurosurg 2000;92(2):235-241

59 Samii M, Gerganov V, Giordano M, Samii A. Two step approach for surgical removal of petroclival meningiomas with large supratentorial extension. Neurosurg Rev 2010;34(2):173-179

60 Samii M, Tatagiba M, Carvalho GA. Resection of large petroclival meningiomas by the simple retrosigmoid route. J Clin Neurosci 1999;6(1):27-30

61 Bambakidis NC, Kakarla UK, Kim LJ, et al. Evolution of surgical approaches in the treatment of petroclival meningiomas: a 
S172 Malignant Tumors of the Clival and Petroclival Region Mohyeldin et al.

retrospective review. Neurosurgery 2007;61(5, Suppl 2):202-209, discussion 209-211

62 Zanation AM, Snyderman CH, Carrau RL, Gardner PA, Prevedello $\mathrm{DM}$, Kassam AB. Endoscopic endonasal surgery for petrous apex lesions. Laryngoscope 2009;119(1):19-25
63 Kassam AB, Gardner P, Snyderman C, Mintz A, Carrau R. Expanded endonasal approach: fully endoscopic, completely transnasal approach to the middle third of the clivus, petrous bone, middle cranial fossa, and infratemporal fossa. Neurosurg Focus 2005; 19(1):E6 